\title{
Open-ended recursive calculation of single residues of response functions for perturbation-dependent basis sets
}

\author{
Daniel H. Friese,* Magnus Ringholm, Bin Gao, and Kenneth Ruud \\ Centre for Theoretical and Computational Chemisty CTCC, Department of Chemistry, \\ University of Tromsø, N-9037 Tromsø, Norway \\ E-mail: daniel.h.friese@uit.no
}

\begin{abstract}
We present theory, implementation and applications of a recursive scheme for the calculation of single residues of response functions which can treat perturbations that affect the basis set. This scheme enables the calculation of non-linear light absorption properties to arbitrary order for other perturbations than the electric field. We apply this scheme for the first treatment of two-photon circular dichroism (TPCD) using London orbitals at the Hartree-Fock level of theory. In general TPCD calculations suffer from the problem of origin dependence which has so far been solved by using the velocity gauge for the electric dipole operator. This work now enables comparison of results from London orbital and velocity gauge based TPCD calculations. We find that the results from the two approaches both exhibit strong basis set dependence but that they are very similar with respect to their basis set convergence.
\end{abstract}

*To whom correspondence should be addressed 


\section{Introduction}

The calculation of single residues of response functions is an important element in the computational treatment of any kind of electronic spectroscopy. Following the pioneering work of Olsen and Jørgensen in $1985,{ }^{1}$ several reformulations have been presented for a wide range of levels of theory. ${ }^{2-6}$

From the single residue of the response functions, different kinds of linear- and non-linear spectroscopic properties can be calculated, such as one-, two- and three-photon absorption, at different levels of theory. ${ }^{7-14}$ As the expressions for higher-order properties can become prohibitively long, we have developed a recursive open-ended response theory code capable of calculating response functions ${ }^{15}$ and the corresponding single residues to arbitrary order ${ }^{5,6}$ but the single residues limited to electric dipole perturbations. We have demonstrated the versatility of the approach by calculating cubic and quartic force fields ${ }^{16}$ and multiphotonabsorption cross sections up to fifth order. ${ }^{6,17}$ Recursive programming has proved to be a very efficient tool for treating these high-order properties: The initial effort invested in making a recursive implementation pays off when considering high-order properties, since it removes the need for the cumbersome theoretical derivations and programming efforts that would otherwise be needed for ad hoc implementations for calculating such properties.

In this work, we will present an extension of the work reported in ref 6 to perturbationdependent basis sets. This will require that we consider the derivatives of the one- and two-electron integrals and the overlap matrix, but also that the perturbation operator is no longer necessarily linear in the field strength as is the case for the electric field.

Perturbation-dependent basis sets are necessary for several properties, such as electronic $^{18-21}$ and two-photon circular dichroism ${ }^{22-24}$ (ECD and TPCD, respectively), which for isotropic samples only are non-vanishing for chiral molecules. These properties describe the differential absorption strengths of one or two circularly polarized photon(s) of different circular polarization. Whereas ECD for isotropic systems depends on the electric and the magnetic dipole operators, TPCD in addition involves the electric quadrupole operator. ${ }^{25,26}$ 
As is the case for all molecular properties that depend on the magnetic dipole operator, calculated ECD and TPCD are in general origin dependent when calculated in a finite basis. For ECD, this problem is normally solved using so-called gauge-including orbitals, also known as London orbitals, ${ }^{27,28}$ in self-consistent field (SCF)-based theory. London orbitals depend explicitly on the magnetic field induction, and are correct to first order in the external field induction for one-electron atomic systems. ${ }^{29}$ In addition to ensuring originindependent results for finite basis sets for variational methods, the use of London orbitals also often leads to faster basis set convergence. ${ }^{30}$ An alternative is to express the electric dipole operator in the velocity gauge. ${ }^{23,31,32}$ This approach gives gauge origin-independent results for non-variational methods, but often at the cost of slower basis set convergence. In the case of ECD, this approach has mainly been used in coupled-cluster theory where the use of London orbitals is not straightforward. ${ }^{33,34}$ In contrast, for TPCD, the velocity gauge formulation is so far the only approach developed in order to obtain origin-independent results in SCF-based theories. This approach was originally introduced by Tinoco in $1975^{25}$ and was rewritten in the framework of response theory by Rizzo et al. in $2006 .{ }^{23}$ The first experimental realization of TPCD appeared in 1995. ${ }^{35}$ Today, most experimental studies of TPCD are assisted by quantum-chemical calculations. ${ }^{36-39}$ London orbitals have neither been used nor assessed in the calculation of two-photon circular dichroism. We here present the first calculations of TPCD in the length gauge using London orbitals.

The rest of this paper is organized as follows: In Section 2, we discuss the general theory of single residues of response functions for perturbation-dependent basis sets. In Section 3, we give an overview of the theory of two-photon circular dichroism, including its origin dependence and its quasi-energy formulation using London orbitals. In Section 4, we discuss the technical details of our calculations before we in Section 5 present the first results of calculations of TPCD using London orbitals, with particular emphasis on the basis-set convergence of the London orbital and velocity gauge approaches. In Section 6 we give some concluding remarks and an outlook. 


\section{Theory: Single residues of response functions for perturbation- dependent basis sets}

In this section, the working equations for the single residues of response functions involving perturbation-dependent basis sets are presented. All expressions can be considered extended versions of the expressions in ref 6 also covering perturbation-dependent basis sets. We will highlight the extension to higher orders through the recursive scheme of refs 6,15 .

\subsection{Density-matrix based response theory}

Our expressions are formulated in the framework of the atomic orbital density-matrix based response theory introduced by Thorvaldsen et al. in $2008,{ }^{5}$ including a general formulation for residue formation. We have already discussed an implementation of this formulation in a recursive scheme for perturbations that do not affect the two-electron integrals (one-electron perturbations) in ref 6 , and we therefore here only briefly recapitulate this theory together with the fundamentals of density-matrix based response theory.

\subsubsection{Energy derivatives in density-matrix based response theory}

As shown by Christiansen, Hättig and Jørgensen in 1998, the time dependence of a perturbed system can be expanded in a Fourier sum of periodic perturbations. ${ }^{2}$ In analogy with the energy of time-independent systems, a so-called time-averaged quasienergy is introduced for the time-dependent systems, which is identical to the energy in the time-independent case. An atomic-orbital density-matrix based quasienergy approach for Kohn-Sham theory was formulated by Thorvaldsen et al. in $2008 .^{5}$

In density-matrix based response theory, the first derivative of the time-dependent quasienergy is the starting point for the perturbation expansion, because the quasienergy itself for sym-

metry reasons cannot be expressed in terms of the density matrix $\tilde{\mathbf{D}}$ and its time derivative $\dot{\tilde{\mathbf{D}}}{ }^{5}$ In the notation we use here, a dot represents a time derivative whereas a tilde denotes 
that the quantity is expressed at a general perturbation field strength which in general is time dependent. The first derivative of the time-averaged quasi-energy is

$$
\tilde{L}^{a}(\tilde{\mathbf{D}})=\tilde{\mathcal{E}}^{0, a}-\tilde{\mathbf{S}}^{a} \tilde{\mathbf{W}}
$$

where $\tilde{\mathcal{E}}^{0, a}$ is the energy differentiated to first order with respect to the perturbation $a$ and to zeroth order with respect to the density matrix, whereas $\tilde{\mathbf{S}}^{a}$ is the perturbed overlap matrix. The generalized energy-weighted density matrix $\tilde{\mathbf{W}}$ is defined as

$$
\tilde{\mathbf{W}}=\tilde{\mathbf{D}} \tilde{\mathcal{F}} \tilde{\mathbf{D}}+\frac{i}{2}(\dot{\tilde{\mathbf{D}}} \tilde{\mathbf{S}} \tilde{\mathbf{D}}-\tilde{\mathbf{D}} \tilde{\mathbf{S}} \dot{\tilde{\mathbf{D}}})
$$

where the Fock matrix $\tilde{\mathbf{F}}$ is given by

$$
\tilde{\mathbf{F}}=\tilde{\mathbf{h}}+\tilde{\mathbf{G}}^{\gamma}(\tilde{\mathbf{D}})+\tilde{\mathbf{V}}^{t}+\tilde{\mathbf{F}}^{x c}-\frac{i}{2} \tilde{\mathbf{T}}
$$

where $\tilde{\mathbf{h}}$ is the one-electron Hamiltonian integrals and $\tilde{\mathbf{G}}^{\gamma}(\tilde{\mathbf{D}})$ the two-electron integrals contracted with the one-electron density matrix $\tilde{\mathbf{D}}$. The superscript $\gamma$ denotes the contribution of exact exchange which depends on the choice of density functional. For pure GGA and LDA functionals, $\gamma$ is zero, for hybrid functionals it is between 0 and 1 and for Hartree-Fock it is 1 . The integrals $\tilde{\mathbf{V}}^{t}$ are the integrals of a one-electron perturbation operator and $\tilde{\mathbf{F}}^{x c}$ is the exchange-correlation contribution to the Fock matrix and is zero in Hartree-Fock (HF) theory. The matrix $\tilde{\mathbf{T}}$ is an anti-Hermitian matrix constructed from combinations of the overlap matrix where the bra or the ket parts have been differentiated with respect to time. ${ }^{5}$

For the formation of response functions, we introduce a time-dependent quasienergy Lagrangian of eq 1 which contains the expression for the time-dependent quasienergy as well as two Lagrangian constraints

$$
\tilde{\mathcal{L}}^{a}=\tilde{L}^{a}(\tilde{\mathbf{D}})-\tilde{\lambda}_{a} \tilde{\mathbf{Y}}-\tilde{\zeta}_{a} \tilde{\mathbf{Z}}
$$


with the Lagrangian multipliers $\tilde{\lambda}_{a}$ and $\tilde{\zeta}_{a}$

$$
\begin{aligned}
& \tilde{\lambda}^{a}=\left[\tilde{\mathbf{D}}^{a} \tilde{\mathbf{S}} \tilde{\mathbf{D}}\right]^{\ominus} \\
& \tilde{\zeta}_{a}=\left[\mathcal{F}^{a}\left(\mathbf{D S}-\frac{1}{2}\right)-\left(\mathbf{F D}-\frac{i}{2} \dot{\tilde{\mathbf{S}}} \tilde{\mathbf{D}}-i \tilde{\mathbf{S}} \dot{\tilde{\mathbf{D}}}\right) \tilde{\mathbf{S}}^{a}\right]^{\oplus}
\end{aligned}
$$

where the notations $\mathbf{A}^{\ominus}$ and $\mathbf{A}^{\oplus}$ were introduced and are given by

$$
\begin{aligned}
& \mathbf{A}^{\ominus}=\mathbf{A}-\mathbf{A}^{\dagger}, \\
& \mathbf{A}^{\oplus}=\mathbf{A}+\mathbf{A}^{\dagger},
\end{aligned}
$$

where adjungation is defined to happen before any differentiation with respect to field strengths. The notation of $\mathbf{F}, \mathbf{D}$ and $\mathbf{S}$ without a tilde denotes quantities evaluated at zero field strength. The matrix $\tilde{\mathbf{Y}}$ in Eq. 4 represents the time-dependent SCF (TDSCF) equation

$$
\tilde{\mathbf{Y}}=\left[\tilde{\mathcal{F}} \tilde{\mathbf{D}} \tilde{\mathbf{S}}-\frac{i}{2} \tilde{\mathbf{S}} \frac{\partial}{\partial t}(\tilde{\mathbf{D}} \tilde{\mathbf{S}})\right]^{\ominus}=\mathbf{0}
$$

Finally, the matrix $\tilde{\mathbf{Z}}$ represents the idempotency relation for the density matrix and is given by

$$
\tilde{\mathbf{Z}}=\tilde{\mathbf{D}} \tilde{\mathbf{S}} \tilde{\mathbf{D}}-\tilde{\mathbf{D}}=\mathbf{0}
$$

Response functions can now be obtained as derivatives of eq. 4 .

As our starting point is the derivative of the time-dependent quasienergy with respect to $a$, the perturbation defined to be perturbation $a$ plays a privileged role in this theory. We therefore have to distinguish between collections of perturbations that involve $a$ and collections that do not involve $a$, as the terms in the quasienergy Lagrangian derivative will not be symmetric with respect to all perturbations. We will therefore use a generalization of 
the well-known $2 m+1$ - and $m+1$-rules of response theory by introducing two parameters $k$ and $n$. These parameters are used to denote the maximum level of differentiation that it is necessary to consider for various constituent terms that are needed to evaluate the differentiated eq 4, notably perturbed density and Fock matrices. Contributions that involve terms perturbed to higher orders than the $k$ and $n$ rules dictate may be truncated and thus need not to be considered. We define that $k$ denotes the maximum level of differentiation needed for constituent terms where the differentiation includes the perturbation defined as $a$, whereas $n$ denotes the same kind of maximum level, but this time for cases where the differentiation does not include perturbation $a$. We have, generally, that $n+k=N-1$ (with $N$ being the total number of perturbations) and we are free to choose an integer $k$ in the interval $[0,(N+1) / 2]($ with $(N+1) / 2$ rounded down for even $N)$.

\subsubsection{Perturbed density matrices}

In contrast to the classical formulation of response functions expressed in terms of molecular orbitals, ${ }^{2}$ the perturbed parameters in density-matrix based response theory are expressed in terms of perturbed density matrices $\mathbf{D}^{K}$, where the superscript $K$ can mean any combination of perturbations. Following the approach in ref 5, the perturbed density matrix can be partitioned as

$$
\mathbf{D}^{K}=\mathbf{D}_{P}^{K}+\mathbf{D}_{H}^{K}
$$

where $\mathbf{D}_{P}^{K}$ and $\mathbf{D}_{H}^{K}$ are referred to as the particular and homogeneous contributions, respectively. The general theory for determining the homogeneous contribution has been presented in refs 5,6 and is therefore not discussed further here.

The general expression for the particular contribution to the perturbed density matrix 
is ${ }^{5}$

$$
\mathbf{D}_{P}^{K}=\mathbf{P} \mathbf{K}^{K-1} \mathbf{P}^{\dagger}-\mathbf{Q} \mathbf{K}^{K-1} \mathbf{Q}^{\dagger}
$$

where we introduced the projection operators

$$
\mathbf{P}=\mathrm{DS}
$$

and

$$
\mathrm{Q}=1-\mathrm{DS}
$$

and the matrix $\mathbf{K}^{K-1}$

$$
\mathbf{K}^{K-1}=-(\mathbf{D S D})_{K-1}^{K},
$$

where the subscript index $K-1$ means that only density matrices perturbed up to one order lower than the order of $K$ are taken into account. Consequently, $\mathbf{D}_{P}^{K}$ contains only contributions involving overlap matrices perturbed up to the order of the perturbation $K$, and density matrices perturbed up to the next order lower than the one of $K$.

Explicit expressions for the particular contribution to the first- and second-order perturbed density matrices are given as

$$
\begin{aligned}
\mathbf{D}_{P}^{b} & =\mathbf{D} \mathbf{S}^{b} \mathbf{D}-\mathbf{D S D S}^{b} \mathbf{D}-\mathbf{D S}^{b} \mathbf{D S D} \\
\mathbf{D}_{P}^{b c} & =\mathbf{D S}(\mathbf{D S})^{b} \mathbf{D}^{c}+\mathbf{D S}(\mathbf{D S})^{c} \mathbf{D}^{b}+\mathbf{D S D}^{b} \mathbf{S}^{c} \mathbf{D}+\mathbf{D S D}^{c} \mathbf{S}^{b} \mathbf{D}+\mathbf{D S D S}^{b c} \mathbf{D}+ \\
& (\mathbf{D S})^{b} \mathbf{D}^{c} \mathbf{S D}+(\mathbf{D S})^{c} \mathbf{D}^{b} \mathbf{S D}+\mathbf{D}^{b} \mathbf{S}^{c} \mathbf{D S D}+\mathbf{D}^{c} \mathbf{S}^{b} \mathbf{D S D}+\mathbf{D S}^{b c} \mathbf{D S D}- \\
& (\mathbf{D S})^{b} \mathbf{D}^{c}-(\mathbf{D S})^{c} \mathbf{D}^{b}-\mathbf{D}^{b} \mathbf{S}^{c} \mathbf{D}-\mathbf{D}^{c} \mathbf{S}^{b} \mathbf{D}-\mathbf{D S}^{b c} \mathbf{D}
\end{aligned}
$$


The rapidly increasing complexity seen in eq 17 demonstrates the benefits of using a recursive approach if even higher-order contributions are to be considered.

\subsection{The formation of residues}

As the general principles of residue formation in atomic orbital density-matrix based response theory have been discussed in refs 5,6 , we refer the reader to the literature for details about residue formation. We will here only recapitulate the general principles in order to define the necessary formalism and notation.

The single residue of a response function $\operatorname{res}(\langle\langle A ; B, C, D\rangle\rangle)$ that depends on the frequencies $\omega_{b}, \omega_{c}$ and $\omega_{d}$, with $-\omega_{a}=\omega_{b}+\omega_{c}+\omega_{d}$, is obtained as

$$
\operatorname{res}(\langle\langle A ; B, C, D\rangle\rangle)=\lim _{\omega_{K} \rightarrow \omega_{q}}\left(\omega_{K}-\omega_{q}\right)\langle\langle A ; B, C, D\rangle\rangle,
$$

where $\omega_{K}$ can either be one of the frequencies $\omega_{a}, \omega_{b}, \omega_{c}$ or $\omega_{d}$ or any sum of $n$ of these frequencies, where $1 \leq n \leq(N-1)$ and $N$ is the number of perturbations. $\omega_{q}$ is an excitation energy of the system. From now on, we will only consider residues which depend on single frequencies, and for the remainder of this work, $K$ will therefore be used as a variable for the index of one perturbation (although the theory is also applicable if $K$ is a combination of different perturbations).

Using the principles shown in refs 5,6 , the formation of the residue in eq 18 can be done using the following simple rules:

1. Remove from the expression for the response function all terms that do not depend on $\mathbf{D}^{K}$ either in the homogeneous or in the particular contribution to a perturbed density matrix.

2. Replace $\mathbf{D}^{K}$ by $\mathbf{D}^{K \rightarrow q}$, where $\mathbf{D}^{K \rightarrow q}$ is defined as

$$
\mathbf{D}^{K \rightarrow q}=\mathbf{D S X}^{K \rightarrow q}-\mathbf{X}^{K \rightarrow q} \mathbf{S D}
$$


where $\mathbf{X}^{K \rightarrow q}$ is the residue of the perturbation parameter matrix corresponding to the excitation into state $q$.

3. For higher-order perturbed density matrices depending on lower-order perturbed density matrices, the expressions have to be simplified in an analogous manner.

A number of terms always vanish from the expression for the response function upon residue formation. In refs 5,6 , it was also shown that the homogeneous part of the perturbed density matrix $\mathbf{D}_{H}^{K}$ can be obtained by scaling the excitation eigenvector $\mathbf{X}_{q}$ by the scalar obtained by contracting the right-hand-side vector $\mathbf{M}_{\mathrm{RHS}}^{K}$ with the adjoint excitation eigenvector $\mathbf{X}_{q}^{\dagger}$

$$
\mathbf{X}^{K \rightarrow q}=-\mathbf{X}_{q} \operatorname{Tr}\left(\mathbf{X}_{q}^{\dagger} \mathbf{M}_{\mathrm{RHS}}^{K}\right)
$$

\subsubsection{The residue of the linear response function}

The linear response function corresponds to the second derivative of the (quasi)energy and therefore, depending on the kind of perturbations applied, describes properties such as molecular Hessians or electric dipole-magnetic dipole polarizabilities (which determines the optical rotation of a system). Its residues are essential for calculating either electronic circular dichroism or first-order non-adiabatic coupling matrix elements. ${ }^{40}$

The residue can be written as

$$
\lim _{\omega_{b} \rightarrow \omega_{q}}\left(\omega_{b}-\omega_{q}\right)\langle\langle A ; B\rangle\rangle \stackrel{\text { Tr }}{=} \mathcal{E}^{1, a} \mathbf{D}^{b \rightarrow q}-\mathbf{S}^{a} \mathbf{W}^{b \rightarrow q}
$$

where the perturbed intermediates

$$
\begin{aligned}
\mathbf{W}^{b \rightarrow q} & =\left[\mathbf{D}^{b \rightarrow q} \mathbf{F D}\right]^{\oplus}+\mathbf{D} \mathcal{F}^{b \rightarrow q} \mathbf{D}+\frac{1}{2} \omega_{q}\left[\mathbf{D}^{b \rightarrow q} \mathbf{S D}\right]^{\ominus} \\
\mathcal{F}^{b \rightarrow q} & =\mathcal{E}^{2}\left(\mathbf{D}^{b \rightarrow q}\right)
\end{aligned}
$$


have been used. The superscript number index on $\mathcal{E}$ denotes derivatives with respect to the density matrix, whereas the superscript letters indicate the perturbations with respect to which $\mathcal{E}$ is differentiated. Note that the perturbed energies $\mathcal{E}^{K \ldots}$ and overlap matrices $\mathbf{S}^{K \ldots}$ are not frequency dependent, in contrast to the perturbed density matrices $\mathbf{D}^{K \ldots}$ and their residues $\mathbf{D}^{K \rightarrow q}$. For brevity, this frequency dependence is not shown explicitly in this work.

\subsubsection{Transition matrix elements}

Eq 20 shows that the solution of the response equations is replaced by the excitation eigenvector (see refs 5,41) multiplied with the scalar $\operatorname{Tr}\left(\mathbf{X}_{q}^{\dagger} \mathbf{M}_{\mathrm{RHS}}^{K}\right)$ when forming the residue. $\mathbf{M}_{\mathrm{RHS}}^{K}$ is obtained from the derivative of the TDSCF equations ${ }^{5,6}$ and depends on the perturbation $K$. Its contraction with the adjoint of the excitation eigenvector is called the right transition matrix element or transition moment $\mathcal{M}_{q \rightarrow 0}^{K}$. There is also a left transition matrix element $\mathcal{M}_{q \leftarrow 0}^{K}$ which can be considered to be the residue of the response function divided by the right transition matrix element. We can therefore write eq 21 as

$$
\lim _{\omega_{b} \rightarrow \omega_{q}}\left(\omega_{b}-\omega_{q}\right)\langle\langle A ; B\rangle\rangle=-\mathcal{M}_{q \leftarrow 0}^{a} \mathcal{M}_{q \rightarrow 0}^{b}
$$

with the two transition matrix elements

$$
\begin{aligned}
\mathcal{M}_{q \rightarrow 0}^{b} & \stackrel{\operatorname{Tr}}{=} \mathbf{X}_{p}^{\dagger} \mathbf{M}_{\mathrm{RHS}}^{b}, \\
\mathcal{M}_{q \leftarrow 0}^{a} & =\operatorname{Tr}\left(\lim _{\omega_{b} \rightarrow \omega_{q}}\left(\omega_{b}-\omega_{q}\right)\langle\langle A ; B\rangle\rangle\right)\left(\mathcal{M}_{q \rightarrow 0}^{b}\right)^{-1} \\
& \stackrel{\operatorname{Tr}}{=} \mathcal{E}^{1, a} \mathbf{D}_{q}-\mathbf{S}^{a} \mathbf{W}^{q}
\end{aligned}
$$

where $\mathbf{D}_{q}$ is the excitation density matrix that can be obtained from eq 19 according to

$$
\mathbf{D}_{q}=\mathbf{D S X}_{q}-\mathbf{X}_{q} \mathbf{S D}
$$


which resembles the original formulation of the residue of the perturbed density matrix (eq 19) where the right transition moment (eq 25) has been factored out. Therefore,

$$
\mathbf{D}^{b \rightarrow q}=-\mathbf{D}_{q} \mathcal{M}_{q \rightarrow 0}^{b}
$$

The right-hand-side vector $\mathbf{M}_{\mathrm{RHS}}^{b}$ in eq 25 determines the inhomogeneous part of the perturbed parameters $\mathbf{X}^{b}$ and is defined when perturbation-dependent basis sets are used as

$$
\mathbf{M}_{\mathrm{RHS}}^{b}=\left[\breve{\mathcal{F}}^{b} \mathbf{D S}+\mathbf{F D}_{P}^{b} \mathbf{S}+\mathbf{F D S}^{b}\right]^{\ominus}-\frac{1}{2} \omega_{b}\left[\mathbf{S D}_{P}^{b} \mathbf{S}+\mathbf{S D S}^{b}\right]^{\oplus},
$$

where $\breve{\mathcal{F}}^{b}$ contains all contributions to $\mathcal{F}^{b}$ which do not depend on the homogeneous contribution to $\mathbf{D}^{b}$. $\mathbf{M}_{\mathrm{RHS}}^{b}$ can be obtained from the TDSCF equation following the procedures

outlined in ref 5. In SCF-based theories, $\mathcal{M}_{q \leftarrow 0}^{b}$ and $\mathcal{M}_{q \rightarrow 0}^{b}$ are related by complex conjugation and therefore only one of them has to be calculated.

The principles outlined here can be applied to higher-order response functions in a similar manner. In the appendix we show this for the quadratic response function.

\subsubsection{Residues of higher-order response functions}

Going beyond the linear response function, it is quickly realized that the expressions rapidly become more complex. The effort required for a tailored formulation of the working equations and its subsequent implementation may quickly become intractable at higher orders, although quadratic and cubic response functions have been derived and implemented using explicit derivation of the working equations. ${ }^{7,42-44}$

To overcome this obstacle, Ringholm, Jonsson and Ruud recently introduced and implemented a recursive scheme for the open-ended treatment of response functions. ${ }^{15}$ This scheme is founded on five algorithms that together allow for the identification and assembly of response functions to arbitrary order. An extension of this scheme, presented in ref 6 , includes the addition of a sixth algorithm, enabling the calculation of single residues for 
electric dipole perturbations. These algorithms work by treating the problem of calculating a response function (or residue) in an order-by-order manner, where, in general terms, each perturbation in turn is allowed to act on the result of having acted with the preceding perturbations on the various quantities necessary for the calculation of the property of interest. The extension to the use of perturbation-dependent basis sets for single residues is straightforward, combining the recursive response theory implementation of ref 15 with the residue formulation described in ref 6 .

\section{Origin independence of chiroptical properties}

The quantum-chemical treatment of chiroptical properties requires the use of the magnetic dipole operator. In a finite basis, the properties calculated with this operator are not origin independent. This problem has received a lot of attention in the literature and different approaches to resolve or reduce this problem have been presented. ${ }^{27,28,45-47}$

The origin dependence of TPCD has been thoroughly discussed by Rizzo and coworkers in 2006 where it was shown that the approach by Tinoco ${ }^{25}$ is origin independent. ${ }^{23}$ This approach is analogous to methods using the velocity gauge for calculating optical rotation or ECD. ${ }^{21,34,48}$

A general two-photon transition strength tensor $\mathcal{W}$ defined with respect to an arbitrary operator $\boldsymbol{w}$ and an electric dipole operator $\boldsymbol{\mu}$ can be written

$$
\mathcal{W}_{\alpha \beta}=\frac{1}{\hbar} \sum_{P} \sum_{n \neq 0} \frac{\left(w_{\alpha}\right)^{0 n}\left(\mu_{\beta}\right)^{n q}}{\omega_{\alpha}-\omega_{n}}
$$

where the summation over $P$ is the summation over all operator-frequency pairs, and $n$ runs over the manifold of excited states. ${ }^{23}$ In this expression, the dipole operator is given in its length-gauge representation. If we now substitute the length representation of the electric dipole operator $\boldsymbol{\mu}$ by its velocity representation $\boldsymbol{\mu}^{p}$, we get, for the relation between the 
different formulations of the tensor, ${ }^{1,23}$

$$
\mathcal{W}_{\alpha \beta}^{p}=-i \omega_{\beta} \mathcal{W}_{\alpha \beta}
$$

where $\mathcal{W}_{\alpha \beta}^{p}$ can be obtained from eq 31 by substituting the dipole operator in length gauge with the dipole operator in velocity gauge, corresponding to the momentum operator, and $\omega_{\beta}$ is the frequency of the photon related to perturbation $\beta$. This relation is discussed in more detail in ref 23 and is based on properties of response functions discussed in ref 1 . Let $\mathbf{H}$ be the electronic Hamiltonian, eq 32 is fulfilled when the commutator relations (in atomic units)

$$
\begin{gathered}
{\left[\mu_{j}, \mathbf{H}\right]=i p_{j},} \\
{\left[\mu_{j}, l_{j}\right]=0}
\end{gathered}
$$

are fulfilled, which is the case for variational methods in an infinite basis. In this equation, $l$ is the angular momentum operator. Most studies of basis set convergence of the length and velocity gauges have been performed for coupled-cluster theory, where velocity gauge based approaches are the only possibility to get origin-independent results. ${ }^{48}$ For optical rotation, the velocity gauge has been shown to display good basis set convergence. ${ }^{32}$ For a comparison of the basis set convergence for the length gauges (with and without London orbitals), see e.g. ref 49. However, coupled-cluster theory in general displays slower basis set convergence than SCF-based approaches due to the handling of electron correlation effects. The 1999 study of ECD by Pedersen, Koch and Ruud also showed that there is a significant dependence on the presence of diffuse basis functions in such coupled-cluster calculations. ${ }^{48}$ An alternative to the velocity gauge is the use of London atomic orbitals ${ }^{27,28}$

$$
\xi_{j}\left(\mathbf{r}_{M}, \mathbf{A}_{M}^{e}\right)=\chi_{j}\left(\mathbf{r}_{M}\right) e^{-i \mathbf{A}_{M}^{e} \mathbf{r}}
$$


where $\chi_{j}$ is a spatial component of an atomic orbital centered on nucleus $M$ with position $\mathbf{R}_{M}$, and where $\mathbf{r}_{M}$ is the difference vector between the position vector of the electron $\mathbf{r}$ and $\mathbf{R}_{M}$. The potential $\mathbf{A}_{M}^{e}$ defines the dependence of the atomic orbital on the external magnetic field strength $\mathbf{B}$ :

$$
\mathbf{A}_{M}^{e}=\frac{1}{2} \mathbf{B} \times \mathbf{R}_{O M} ; \quad \mathbf{R}_{O M}=\mathbf{R}_{M}-\mathbf{O}
$$

where $\mathbf{O}$ is the global gauge origin.

\section{Computational details}

\subsection{Molecules and structures}

We have calculated the two-photon circular dichroism for three chiral molecules: hydrogen peroxide, propylene oxide and $R$-1,1'-bi(2-naphthol) (R-BINOL). Hydrogen peroxide was studied by Rizzo et al., investigating the origin dependence of TPCD. ${ }^{23}$ For hydrogen peroxide we used the same frozen chiral geometry as in their work. For propylene oxide, we used the structure by Srebro et al., ${ }^{50}$ optimized at the B3LYP level ${ }^{51}$ with the 6-311G(d,p) basis set. ${ }^{52,53}$ Finally, as a large-scale application, we consider R-BINOL using the geometry of ref 36 .

\subsection{TPCD calculations}

All TPCD calculations have been performed using the augmented Dunning-style basis sets aug-cc-pVDZ, aug-cc-pVTZ, aug-cc-pVQZ and aug-cc-pV5Z ${ }^{54,55}$ as well as their doubly aug-

mented analogues. Both the velocity gauge approach by Tinoco ${ }^{23,25}$ and the length-gauge approach (referred to as the $\omega_{0}$ approach in ref 23) have been used for the magnetic dipole operator. To ensure origin independence of the results in the length-gauge approach, London atomic orbitals have been used. All calculations have been carried out at the Hartree-Fock 
level of theory. While the length gauge calculations have been carried out using the code outlined earlier as a submodule of the DALTON program, the velocity-based calculations were done using DALTON. ${ }^{56}$

The two-photon circular dichroism $\sigma^{\text {TPCD }}$ measures the differential two-photon absorption cross sections for left and right circularly polarized photons and is obtained from the equation $^{23}$

$$
\sigma^{\mathrm{TPCD}}=\sigma_{\mathrm{L}}^{2 \mathrm{P}}-\sigma_{\mathrm{R}}^{2 \mathrm{P}}=\frac{4}{15} \frac{4 \pi^{2} N_{A} \omega^{2} g(2 \omega)}{c_{0}^{3}\left(4 \pi \epsilon_{0}\right)^{2}} \cdot{ }^{f} R^{\mathrm{TPCD}}
$$

where $N_{A}$ is Avogadro's number, $\omega$ is the energy of the incident photons (half the excitation energy in this work) and $g(2 \omega)$ is the lineshape function, $c_{0}$ is the speed of light in vacuo and $\epsilon_{0}$ is the electric constant. In this work, we use a Lorentzian lineshape function with a full-width of $0.1 \mathrm{eV}$ at half maximum. The Lorentzian is centered at the excitation energy

of the state considered. The quantity ${ }^{f} R^{\mathrm{TPCD}}$ is the two-photon rotatory strength. It is important to note that eq 37 is the same for both approaches used in this work, and that the methods only differ in the determination of ${ }^{f} R^{\mathrm{TPCD}}$.

\subsubsection{Rotatory strength in the Tinoco approach}

The rotatory strength is obtained from ${ }^{23,25,57}$

$$
{ }^{f} R^{\mathrm{TPCD}, \mathrm{TI}}=-b_{1} \mathcal{B}_{1}-b_{2} \mathcal{B}_{2}-b_{3} \mathcal{B}_{3},
$$

where $b_{1}, b_{2}$ and $b_{3}$ are coefficients depending on the propagation direction and polarization of the incident photons. In this study, we investigate TPCD as a difference between the absorption cross sections for two left and two right circularly polarized photons propagating parallel to each other and orthogonal to the polarization plane, and for this setup $b_{1}=6$, 
$b_{2}=2$, and $b_{3}=-2 .{ }^{23,25}$ The three transition strength contributions are defined as

$$
\begin{aligned}
\mathcal{B}_{1} & =\frac{1}{\omega^{3}} \sum_{a b} M_{a b}^{p, 0 q} P_{a b}^{q 0} \\
\mathcal{B}_{2} & =\frac{1}{2 \omega^{3}} \sum_{a b} T_{a b}^{+, 0 q} P_{a b}^{q 0} \\
\mathcal{B}_{3} & =\frac{1}{\omega^{3}} \sum_{a b} M_{a a}^{p, 0 q} P_{b b}^{q 0}
\end{aligned}
$$

where $\mathbf{M}^{p, 0 q}$ and $\mathbf{P}^{0 q}$ are the electric dipole-magnetic dipole transition moment tensor and the electric dipole-electric dipole transition moment tensor, respectively with the electric dipole operator expressed in in velocity gauge. In the sum-over-states approach, they can be expressed as

$$
M_{a b}^{p, 0 q}=\frac{1}{\hbar} \sum_{P} \sum_{n \neq 0} \frac{m_{a}^{0 n} \mu_{b}^{p, n q}}{\omega_{a}-\omega_{q}}, \quad P_{a b}^{0 q}=\frac{1}{\hbar} \sum_{P} \sum_{n \neq 0} \frac{\mu_{a}^{p, 0 n} \mu_{b}^{p, n q}}{\omega_{a}-\omega_{q}}
$$

where $\boldsymbol{m}$ is the magnetic dipole operator. The summation $\sum_{P}$ is a sum over all permutations of the perturbations $a$ and $b$ and their frequencies. The elements of the tensor $\mathbf{T}^{+, 0 q}$ are obtained from the electric dipole-electric quadrupole transition moment tensor according to

$$
T_{a b}^{+, 0 q}=\frac{1}{\hbar} \epsilon_{b c d} \sum_{P} \sum_{n \neq 0} \frac{T_{a c}^{0 n} \mu_{d}^{p, n q}}{\omega_{a}-\omega_{f}}
$$

where $\epsilon_{b c d}$ is the Levi-Civita antisymmetric tensor and the matrix elements of the electric quadrupole operator in velocity gauge are defined using atomic units as

$$
T_{a b}=p_{a} r_{b}+p_{b} r_{a}
$$

where $p$ and $r$ are matrix elements of the momentum and length operators, respectively. Note that excitation strength tensors are marked with the superscript index $0 q$ whereas deexcitation tensors are marked with $q 0$. These two kinds of tensors are connected by 
complex conjugation in the SCF-based theory we are considering in this work.

\subsubsection{Rotatory strength in London orbitals/length gauge approach}

The London orbitals/length gauge (LL) approach we use in this work is based on the length gauge $\omega_{0}$ approach discussed in ref 23 . In contrast to the approach in ref 23 , we use London orbitals for the treatment of the magnetic dipole operator. In both the $\omega_{0}$ and the LL approach, the rotatory strength is therefore obtained as

$$
{ }^{f} R^{\mathrm{TPCD}, \mathrm{LO}}=-i b_{1} \mathcal{B}_{1}+b_{2} \mathcal{B}_{2}-i b_{3} \mathcal{B}_{3}
$$

which looks very similar to eq 38 with the exception of some signs and the imaginary unit $i$ related to the imaginary character of the magnetic dipole operator. The coefficients $b_{1}, b_{2}$ and $b_{3}$ are chosen in the same way as for the Tinoco approach. The contributions are defined as

$$
\begin{aligned}
\mathcal{B}_{1} & =\sum_{a b} M_{a b}^{0 q} S_{a b}^{q 0} \\
\mathcal{B}_{2} & =\frac{\omega}{2} \sum_{a b} Q_{a b}^{+, 0 q} S_{a b}^{q 0} \\
\mathcal{B}_{3} & =\sum_{a b} M_{a a}^{0 q} S_{b b}^{q 0}
\end{aligned}
$$

where $\mathbf{M}^{0 q}$ is the electric dipole-magnetic dipole transition strength tensor and $\mathbf{S}^{f 0}$ is the electric dipole-electric dipole transition strength tensor, constructed according to

$$
\begin{aligned}
M_{a b}^{0 q} & =\frac{1}{\hbar} \sum_{P} \sum_{n \neq 0} \frac{m_{a}^{0 n} \mu_{b}^{n q}}{\omega_{a}-\omega_{q}} \\
S_{a b}^{0 q} & =\frac{1}{\hbar} \sum_{P} \sum_{n \neq 0} \frac{\mu_{a}^{0 n} \mu_{b}^{n q}}{\omega_{a}-\omega_{q}} .
\end{aligned}
$$


In contrast to the Tinoco approach, the dipole operator $\boldsymbol{\mu}$ is here expressed in the length gauge. The elements of the transition-strength tensor $\mathbf{Q}^{+, 0 q}$ are obtained from the electric dipole and electric quadrupole operators, both expressed in the length gauge as

$$
Q_{a b}^{+, 0 q}=\frac{1}{\hbar} \epsilon_{b c d} \sum_{P} \sum_{n \neq 0} \frac{\mathcal{Q}_{a c}^{0 n} \mu_{d}^{n q}}{\omega_{a}-\omega_{q}} .
$$

The operator $\mathcal{Q}$ is the traced form of the quadrupole operator, which in atomic units is defined as

$$
\mathcal{Q}=\mathbf{r r}^{T}
$$

where $\mathbf{r}$ is the length operator.

As shown in ref 23, the contributions $\mathcal{B}_{1}$ and $\mathcal{B}_{3}$ are origin dependent, whereas $\mathcal{B}_{2}$ is origin independent by construction. The problem of origin dependence is solved by using London orbitals for the electric dipole-magnetic dipole transition moment tensor, ${ }^{58}$ which is then used to calculate both $\mathcal{B}_{1}$ and $\mathcal{B}_{3}$.

\section{Applications}

In this section, we will compare the basis set dependence of the different approaches for the calculation of TPCD. As we are not comparing with experimental results, we do not focus on the complete TPCD rotatory strength, but rather on the different contributions in order to explore whether they behave differently in the different approaches.

\subsection{TPCD of hydrogen peroxide}

For hydrogen peroxide, we studied the lowest-energy state that has $\pi^{*} \rightarrow \sigma^{*}$ character. The occupied orbital is the $\pi^{*}$-antibonding orbital between the two oxygen atoms, whereas the virtual orbital is the corresponding $\sigma^{*} \mathrm{O}-\mathrm{O}$ orbital. 
Our results for hydrogen peroxide are listed in Table 1. Plots for the three contributions are shown in Figure 1. From these results, we note that the different contributions depend strongly on the basis set and that the basis-set convergence differs for the two approaches used. In general, basis-set convergence is faster for the length gauge. $\mathcal{B}_{1}$ can be considered converged already at the aug-cc-pVDZ level in both approaches, whereas $\mathcal{B}_{3}$ requires at least aug-cc-pVQZ for converged results. The results obtained with doubly augmented basis sets are very similar to the ones for the singly augmented ones.

$\mathcal{B}_{2}$ shows the largest deviations for small basis sets. However, with the LL approach and the aug-cc-pVQZ basis set, the contribution can for both approaches be considered to be converged with respect to the basis set. Compared to the other two contributions, $\mathcal{B}_{2}$ is quite small and its influence on the rotatory strength is therefore modest. In general, we have to distinguish between convergence with respect to the basis and the agreement between the two approaches. The data for the three contributions show that convergence with respect to the basis set is reached for the aug-cc-pVDZ basis set in the LL approach, whereas the Tinoco approach requires at least an aug-cc-pVTZ $\left(\mathcal{B}_{1}, \mathcal{B}_{2}\right)$ or aug-cc-pVQZ $\left(\mathcal{B}_{3}\right)$ basis set, respectively, to reach convergence. Also this contribution shows nearly the same behaviour with doubly augmented basis sets.

As for the intermediate quantities, the basis set convergence of ${ }^{f} R^{\mathrm{TPCD}}$ is faster for the LL approach. It is worth noting that the aug-cc-pVDZ basis set shows notable deficiencies in the description of all contributions to the rotatory strength in the Tinoco approach, as well as for the rotatory strength itself.

For hydrogen peroxide we also studied the origin dependence of the length gauge approach. We used three shifted geometries of the hydrogen peroxide molecule, one in which the origin was shifted to one of the hydrogen atoms (in the original geometry it is on one of the oxygen atoms), one in which the $y$ component of the Cartesian coordinates was shifted by 5 a.u. and one in which the shift in the $y$ coordinates was 100 atomic units. Calculations have been performed using the aug-cc-pVDZ basis set. The results are listed in Table 2. These 
Table 1: TPCD and the different contributions for hydrogen peroxide for different basis sets. $\Delta E$ is the excitation energy.

\begin{tabular}{cccccccccc}
\hline \hline & & \multicolumn{2}{c}{$\mathcal{B}_{1}$} & \multicolumn{2}{c}{$\mathcal{B}_{2}$} & \multicolumn{2}{c}{$\mathcal{B}_{3}$} & \multicolumn{2}{c}{$R^{\mathrm{TPCD}}$} \\
\hline & $\Delta E$ & LL & Tinoco & LL & Tinoco & LL & Tinoco & LL & Tinoco \\
& {$[$ a.u.] } & {$[$ a.u.] } & {$[$ a.u.] } & {$[$ a.u.] } & {$[$ a.u.] } & {$[$ a.u.] } & {$[$ a.u.] } & {$[$ a.u.] } & {$[$ a.u.] } \\
\hline aug-cc-pVDZ & 0.21336 & -1.608 & -0.232 & 0.033 & -1.629 & -1.505 & -7.694 & 6.702 & -17.254 \\
aug-cc-pVTZ & 0.21665 & -1.644 & -1.792 & 0.031 & -0.097 & -1.597 & -2.932 & 6.731 & 4.698 \\
aug-cc-pVQZ & 0.21687 & -1.696 & -1.629 & 0.027 & -0.009 & -1.633 & -1.844 & 6.963 & 6.071 \\
aug-cc-pV5Z & 0.21693 & -1.713 & -1.669 & 0.027 & -0.004 & -1.650 & -1.734 & 7.031 & 6.536 \\
& & & & & & & & & \\
d-aug-cc-pVDZ & 0.21350 & -1.686 & 1.109 & 0.023 & -1.460 & -1.604 & -7.038 & 6.953 & -23.648 \\
d-aug-cc-pVTZ & 0.21671 & -1.732 & -1.614 & 0.022 & 0.015 & -1.658 & -2.617 & 7.118 & 4.483 \\
d-aug-cc-pVQZ & 0.21689 & -1.727 & -1.568 & 0.022 & 0.021 & -1.660 & -1.766 & 7.087 & 5.916 \\
d-aug-cc-pV5Z & 0.21693 & -1.725 & -1.665 & 0.022 & 0.021 & -1.660 & -1.718 & 7.076 & 6.594 \\
\hline \hline
\end{tabular}
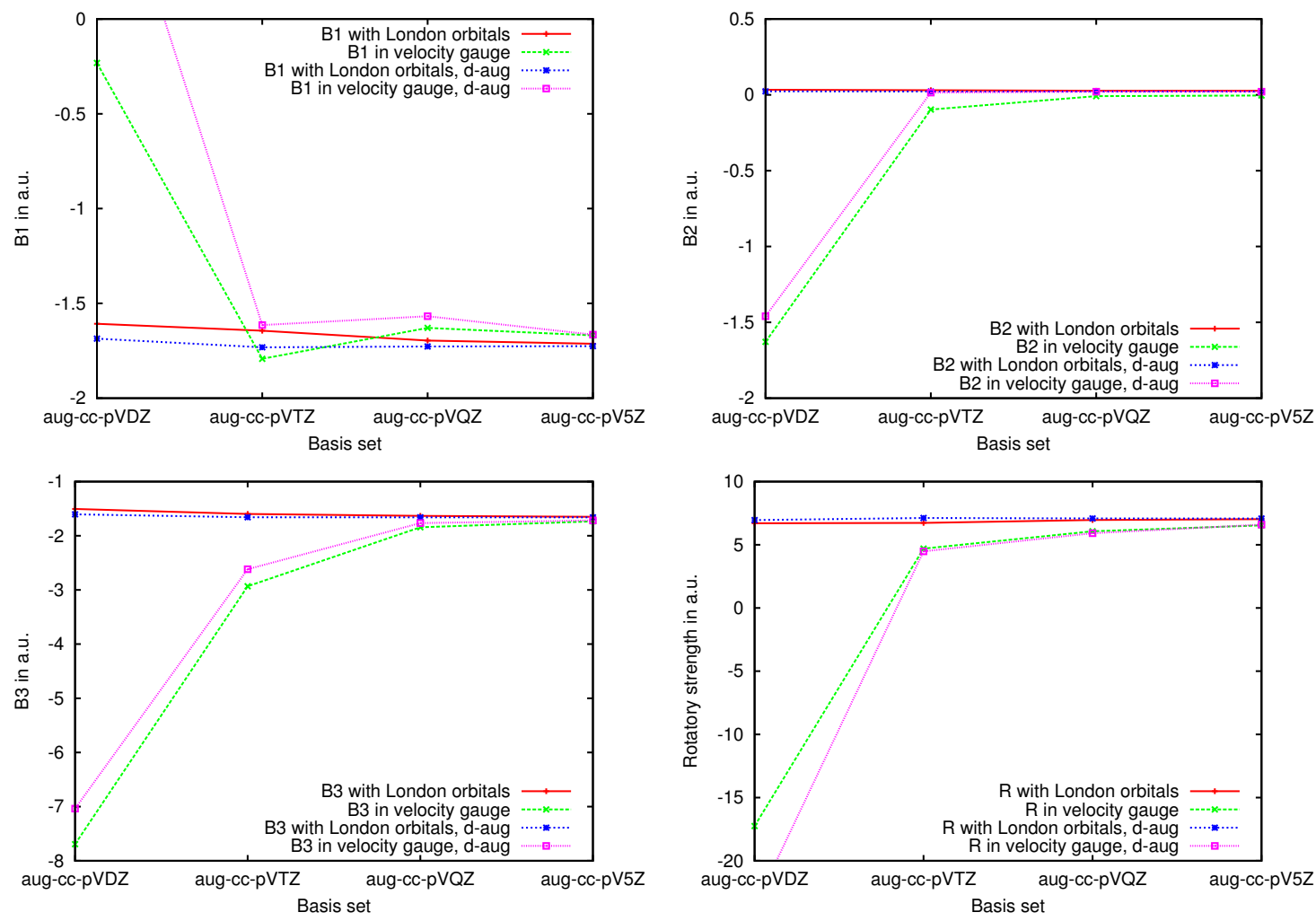

Figure 1: Plotted results for the different contributions $\mathcal{B}_{1}$ (top left), $\mathcal{B}_{2}$ (top right) and $\mathcal{B}_{3}$ (bottom left) to the TPCD rotatory strength ${ }^{f} R^{\mathrm{TPCD}}$ (bottom right) of hydrogen peroxide. 
Table 2: Comparison between shifted and non-shifted geometries for hydrogen peroxide. The columns marked LL contain the results from our London-orbital length gauge approach while the numbers in columns marked $\omega_{0}$ were obtained using the complete length-gauge approach without London orbitals reported by Rizzo et al. ${ }^{23}$

\begin{tabular}{ccccccccc}
\hline \hline & \multicolumn{2}{c}{ non-shifted } & \multicolumn{2}{c}{ shifted on H } & \multicolumn{2}{c}{ shifted, 5 a.u. in $y$} & \multicolumn{2}{c}{ shifted, 100 a.u. in $y$} \\
\hline & LL & $\omega_{0}$ & LL & $\omega_{0}$ & LL & $\omega_{0}$ & LL & $\omega_{0}$ \\
& [a.u.] & [a.u.] & [a.u.] & [a.u.] & [a.u.] & [a.u.] & [a.u.] & [a.u.] \\
\hline $\mathcal{B}_{1}$ & -1.6076 & -1.6284 & -1.6076 & -1.6348 & -1.6076 & -1.5674 & -1.6075 & -4.0785 \\
$\mathcal{B}_{2}$ & 0.0333 & 0.0333 & 0.0333 & 0.0333 & 0.0333 & 0.0333 & 0.0328 & 0.0333 \\
$\mathcal{B}_{3}$ & -1.5050 & -1.5092 & -1.5050 & -1.5354 & -1.5050 & -1.2562 & -1.5050 & 3.5519 \\
\hline${ }^{f} R$ & 6.7021 & 6.8188 & 6.7021 & 6.8043 & 6.7020 & 6.9587 & 6.7006 & 31.6415 \\
\hline \hline
\end{tabular}

results illustrate that there is a significant difference between the LL- and the $\omega_{0}$ results in general, but that this is very moderate for the non-shifted geometry and for the one with the origin at one of the hydrogen atoms. For the geometries with larger shifts, the differences become more significant and we note that for the results with the shift of 100 a.u. even the results with the London orbitals show a small but visible deviation from the results for the more moderate shifts due to numerical errors arising from the finite convergence threshold used when determining the response vectors. However, we note a strong origin dependence on the results from the $\omega_{0}$ approach for the strongly shifted geometries.

\section{$5.2 \quad$ TPCD of propylene oxide}

For propylene oxide, we investigated the TPCD of the $S_{1}$ state which is dominated by the transition from the highest occupied molecular orbital to the lowest unoccupied molecular orbital. The state is of Rydberg character.

Our results for the propylene oxide molecule are collected in Table 3 and plotted in Figure 2. Comparing them with the results for hydrogen peroxide, we note that for the two magnetic contributions, the two approaches give similar results already for aug-cc-pVDZ, but that basis set convergence requires a basis set of at least aug-cc-pVTZ quality.

For the quadrupole term $\mathcal{B}_{2}$, basis set convergence of the Tinoco approach is somewhat 
faster than for the LL approach, but the basis set dependence of this term is in general smaller than that of the magnetic terms. The rotatory strength is converged both with respect to the basis set and the approach with the aug-cc-pVTZ basis set.

Table 3: TPCD and the different contributions for propylene oxide for different basis sets. $\Delta E$ is the excitation energy.

\begin{tabular}{cccccccccc}
\hline \hline & $\Delta E$ & \multicolumn{2}{c}{$\mathcal{B}_{1}$} & \multicolumn{2}{c}{$\mathcal{B}_{2}$} & \multicolumn{2}{c}{$\mathcal{B}_{3}$} & \multicolumn{2}{c}{$R^{\mathrm{TPCD}}$} \\
\hline & & $\mathrm{LL}$ & Tinoco & LL & Tinoco & LL & Tinoco & LL & Tinoco \\
& [a.u.] & [a.u.] & [a.u.] & [a.u.] & [a.u.] & [a.u.] & [a.u.] & {$[$ a.u.] } & [a.u.] \\
\hline aug-cc-pVDZ & 0.32481 & -3.799 & -3.636 & 2.907 & 2.425 & -8.513 & -8.153 & 11.580 & 10.362 \\
aug-cc-pVTZ & 0.32444 & -3.103 & -3.153 & 2.659 & 2.205 & -7.520 & -7.338 & 8.898 & 8.653 \\
aug-cc-pVQZ & 0.32434 & -3.052 & -3.052 & 2.489 & 2.237 & -7.214 & -7.184 & 8.862 & 8.421 \\
aug-cc-pV5Z & 0.32418 & -2.996 & -2.981 & 2.399 & 2.284 & -7.091 & -7.049 & 8.591 & 8.358 \\
\hline \hline
\end{tabular}

\subsection{The R-BINOL molecule}

The R-BINOL molecule has $C_{2}$-symmetry. This has special implications for the evaluation of the TPCD. In this work, the $1 B$ state is considered, which is the lowest excited state for all three basis sets used. The results are tabulated in Table 4. Note that there is no $\mathcal{B}_{3}$ contribution to the TPCD rotatory strength for this state as the diagonal elements of both the electric dipole-electric dipole and electric dipole-magnetic dipole transition moment tensors are zero. The structure of the R-BINOL molecule is shown in Figure 3.

The results show that the contribution of $\mathcal{B}_{1}$ is again significantly larger than $\mathcal{B}_{2}$. In contrast to the propylene oxide molecule, the basis set dependence of $\mathcal{B}_{1}$ is significantly larger than that of $\mathcal{B}_{2}$. Comparing the two approaches, we note that there are significant differences for the $\mathcal{B}_{2}$ contribution, whereas for the $\mathcal{B}_{1}$ contributions, both approaches yield rather similar results.

The difference between the methods is not only significant for the $\mathcal{B}_{2}$ contribution, but also for the rotatory strength which only for the aug-cc-pVTZ basis set displays convergence of the two approaches. The difference in the two approaches for the rotatory strength is still 

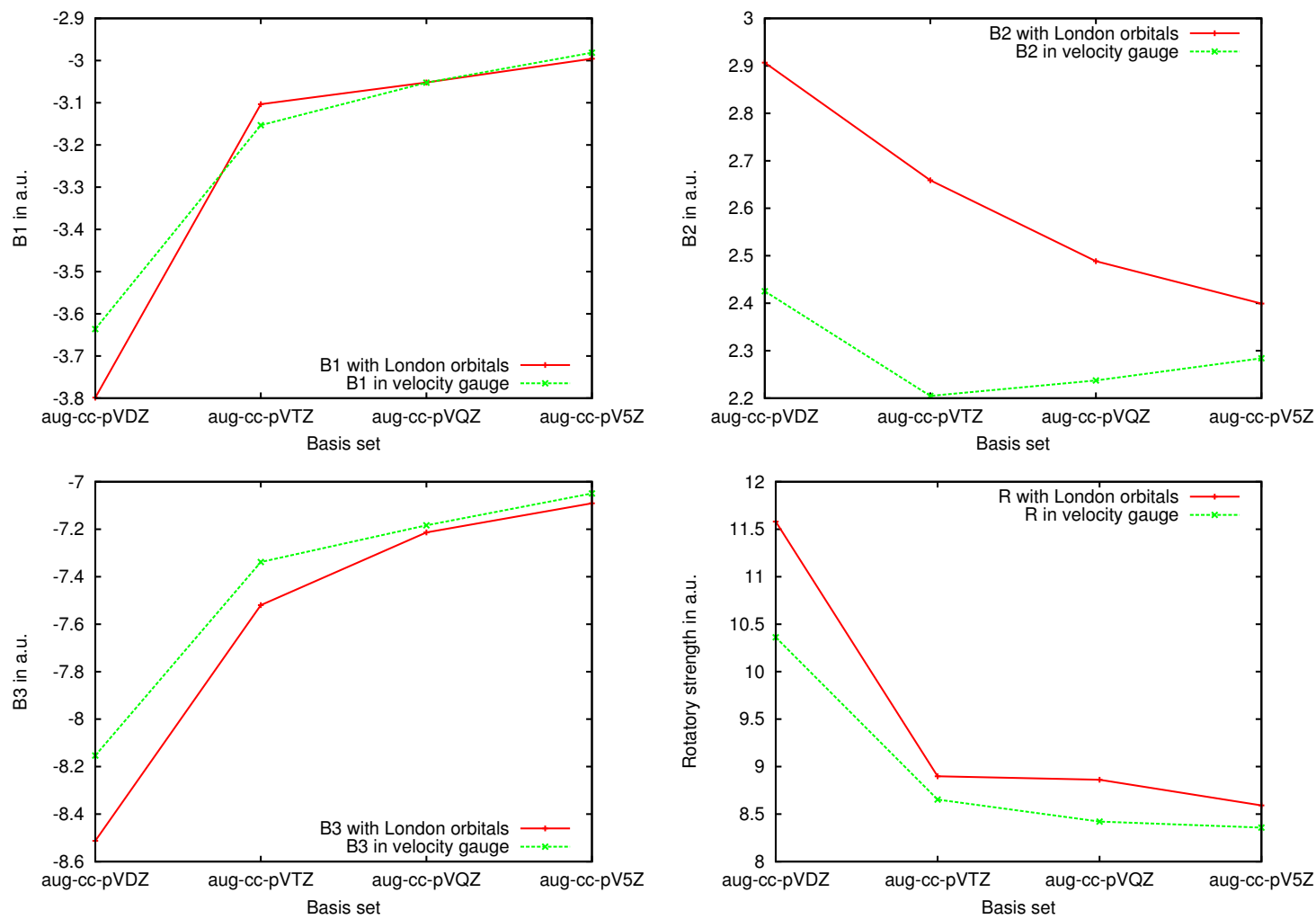

Figure 2: Plotted results for the different contributions $\mathcal{B}_{1}$ (top left), $\mathcal{B}_{2}$ (top right) and $\mathcal{B}_{3}$ (bottom left) to the TPCD rotatory strength (bottom right) of propylene oxide. 


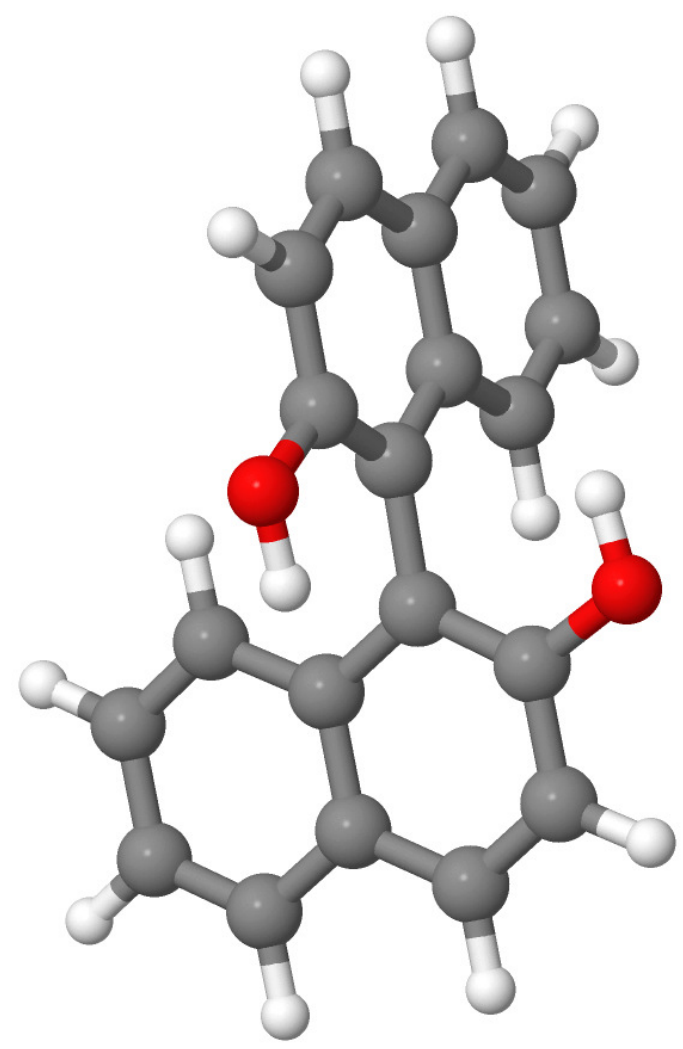

Figure 3: Molecular structure of R-BINOL. 
significant for the aug-cc-pVDZ basis set, with the results from the Tinoco approach being closer to the ones for the larger basis sets in this case.

Table 4: TPCD and the different contributions for R-BINOL for different basis sets. The $\mathcal{B}_{3}$ contribution is not listed as it is zero for symmetry reasons. $\Delta E$ is the excitation energy.

\begin{tabular}{cccccccc}
\hline \hline & $\Delta E$ & \multicolumn{2}{c}{$\mathcal{B}_{1}$} & \multicolumn{2}{c}{$\mathcal{B}_{2}$} & \multicolumn{2}{c}{$R^{\mathrm{TPCD}}$} \\
\hline & & LL & Tinoco & LL & Tinoco & LL & Tinoco \\
& [a.u.] & [a.u.] & [a.u.] & [a.u.] & [a.u.] & [a.u.] & [a.u.] \\
\hline aug-cc-pVDZ & 0.17038 & -5.657 & -5.739 & -0.038 & 2.610 & 33.865 & 39.652 \\
aug-cc-pVTZ & 0.16982 & -6.222 & -6.306 & 0.051 & 0.360 & 37.437 & 38.556 \\
\hline \hline
\end{tabular}

\section{Conclusion and Outlook}

We have presented an open-ended recursive approach for the calculation of single residues of response functions to arbitrary order for perturbation-dependent basis sets, allowing for studies of both magnetic-field perturbations using London orbitals and geometrical distortions. We have used the implementation to present the first calculations of TPCD using London atomic orbitals, allowing for gauge-origin independent results also in the length gauge. We have compared the use of London orbitals in the length gauge with the conventional velocitygauge approach, showing that the two approaches converge to the same limit when increasing the size of the basis set. There is not a uniform basis set convergence behaviour for the different contributions to the TPCD rotatory strength. Whereas the magnetic field contributions converge quite well in most cases, the quadrupole component (which does not require the use of London orbitals) is quite challenging. Convergence with respect to the approach is only achieved for basis sets of at least aug-cc-pVTZ size. In the case of propylene oxide, the quadrupole contribution required even larger basis sets for convergence. This, however, did not affect the results for the rotatory strength significantly. Double augmentation of the basis sets does not seem to change this remarkably. For hydrogen peroxide, we showed that the regular length gauge is origin dependent, but that the London orbitals can rectify 
this to within the numerical accuracy of the results determined by the finite threshold of convergence of the response equations.

Our study has shown that the use of London orbitals in the length-gauge formulation of TPCD allows for origin-independent rotatory strengths. We have also demonstrated that the LL approach does not lead to significantly faster basis set convergence than the results obtained with the velocity gauge, lending support to the quality of the results presented in the literature to date. Nevertheless, it may be worthwhile to explore the basis set convergence of the two approaches also for somewhat larger molecules than studied here. At present this methodology is only available at the Hartree-Fock level of theory, but the implementation of a DFT version is in progress.

This study is an extension of our previous work on the use of London atomic orbitals for calculating different nonlinear optical birefringences and dichroisms, ${ }^{59}$ such as Buckingham birefringence, ${ }^{60,61}$ the Cotton-Mouton effect, ${ }^{62}$ Jones birefringence ${ }^{63}$ and magnetic circular dichroism. ${ }^{64}$ Our present study is the second example of the use of London atomic orbitals in the calculation of single residues of a nonlinear response function, but in contrast to the case of magnetic circular dichroism for which the magnetic field is static, the magnetic-field component is frequency dependent in the case of TPCD.

The great diversity in linear and nonlinear properties that can be calculated using London orbitals is now further accentuated through the use of recursive programming techniques. ${ }^{6,15}$ This demonstrates the versatility of London atomic orbitals as a means to achieve originindependent molecular properties. In many cases, the use of London atomic orbitals also leads to faster basis set convergence ${ }^{63,65}$ However, this is not universal, and for properties such as optical rotation ${ }^{66}$ and the Cotton-Mouton effect, ${ }^{62}$ there is not any significant improvement in basis set convergence from the use of London atomic orbitals except for very small basis sets. The results presented here for TPCD also suggest that faster basis set convergence is not guaranteed, although the results are not conclusive. Nevertheless, our work represents an important step towards a general approach for calculating gauge-origin inde- 
pendent transition moments and excited-state birefringences and dichroisms using London atomic orbitals.

\section{Acknowledgements}

The authors acknowledge support by Radovan Bast, Stockholm, in debugging the parallel London orbital code. This work has received support from the Research Council of Norway through a Centre of Excellence Grant (Grant No. 179568/V30), from the European Research Council through a Starting Grant (Grant No. 279619) and from from the Norwegian Supercomputing Program (Grant No. NN4654K).

\section{A Residues of the quadratic response function}

The quadratic response function using $k=1$ and $n=1$ (see Section 2.1.1) is

$$
\langle\langle A ; B, C\rangle\rangle_{\omega_{b}, \omega_{c}} \stackrel{T r}{=} \mathcal{E}_{1,1}^{a b c}-(\mathbf{S W})_{(1,1)_{W}}^{a b c}-\mathbf{S}^{a} \mathbf{W}_{1^{\prime}}^{b c}-\lambda_{a} \mathbf{Y}_{1^{\prime}}^{b c}-\zeta_{a} \mathbf{Z}_{1^{\prime}}^{b c}
$$


where $1^{\prime}$ denotes that the corresponding intermediates only contain perturbed parameters (i.e. Fock, density, and overlap matrices) up to first order. The intermediate quantities are

$$
\begin{aligned}
\mathcal{E}_{1,1}^{a b c}= & \mathcal{E}^{0, a b c}+\mathcal{E}^{1, a b} \mathbf{D}^{c}+\mathcal{E}^{1, a c} \mathbf{D}^{b}+\mathcal{E}^{2, a}\left(\mathbf{D}^{b}\right) \mathbf{D}^{c}+\mathcal{F}_{1}^{b c} \mathbf{D}^{a} \\
\mathcal{F}_{1}^{b c}= & \mathcal{E}^{1, b c}+\mathcal{E}^{2, b}\left(\mathbf{D}^{c}\right)+\mathcal{E}^{2, c}\left(\mathbf{D}^{b}\right)+\mathcal{E}^{3}\left(\mathbf{D}^{b}, \mathbf{D}^{c}\right) \\
(\mathbf{S W})_{(1,1)_{W}}^{a b c}= & \mathbf{S}^{a b c} \mathbf{W}+\mathbf{S}^{a b} \mathbf{W}^{c}+\mathbf{S}^{a c} \mathbf{W}^{b}+\mathbf{S}^{b c} \mathbf{W}^{a} \\
\mathbf{W}_{1^{\prime}}^{b c}= & {\left[\mathbf{D}^{b} \mathcal{F}^{c} \mathbf{D}+\mathbf{D}^{b} \mathcal{F} \mathbf{D}^{c}+\mathbf{D}^{c} \mathcal{F}^{b} \mathbf{D}\right]^{\oplus}+} \\
& \frac{1}{2}\left[\omega_{b}\left(\mathbf{D}^{b} \boldsymbol{S}^{c} \mathbf{D}+\mathbf{D}^{b} \boldsymbol{S} \mathbf{D}^{c}\right)+\omega_{c}\left(\mathbf{D}^{c} \boldsymbol{S}^{b} \mathbf{D}+\mathbf{D}^{c} \boldsymbol{S} \mathbf{D}^{b}\right)\right] \\
\mathbf{Y}_{1^{\prime}}^{b c}= & {\left[\mathcal{F}^{b}\left(\mathbf{D}^{c} \mathbf{S}+\mathbf{D} \mathbf{S}^{c}\right)+\mathcal{F}^{c}\left(\mathbf{D}^{b} \mathbf{S}+\mathbf{D} \mathbf{S}^{b}\right)+\mathbf{F}\left(\mathbf{D}^{b} \mathbf{S}^{c}+\mathbf{D}^{c} \mathbf{S}^{b}\right)\right] } \\
& \frac{1}{2}\left[\omega_{b} \mathbf{S}^{c}\left(\mathbf{D}^{b} \mathbf{S}+\mathbf{D S}^{b}\right)+\omega_{c} \mathbf{S}^{b}\left(\mathbf{D}^{c} \mathbf{S}+\mathbf{D} \mathbf{S}^{c}\right)\right]^{\oplus}+\frac{1}{2}\left(\omega_{b}+\omega_{c}\right)\left(\left[\mathbf{S}\left(\mathbf{D}^{b} \mathbf{S}^{c}+\mathbf{D}^{c} \mathbf{S}^{b}\right)\right]^{\oplus}\right), \\
\mathbf{Z}_{1^{\prime}}^{b c}= & {\left[\mathbf{D}^{b} \boldsymbol{S}^{c} \mathbf{D}+\mathbf{D}^{b} \mathbf{S} \mathbf{D}^{c}+\mathbf{D}^{c} \boldsymbol{S}^{b} \mathbf{D}\right]^{\oplus} . }
\end{aligned}
$$

The residue for the response function in eq 53 is

$$
\begin{aligned}
\lim _{\omega_{c} \rightarrow \omega_{q}}\left(\omega_{c}-\omega_{q}\right)\langle\langle A ; B, C\rangle\rangle_{\omega_{b}, \omega_{c}} \stackrel{T r}{=} & \mathcal{E}^{1, a b} \mathbf{D}^{c \rightarrow q}+\mathcal{E}^{2, a}\left(\mathbf{D}^{b}\right) \mathbf{D}^{c \rightarrow q}+ \\
& \mathcal{F}_{1}^{b(c \rightarrow q)} \mathbf{D}^{a}-\mathbf{S}^{a b} \mathbf{W}^{c \rightarrow q}-\mathbf{S}^{a} \mathbf{W}_{1^{\prime}}^{b(c \rightarrow q)}- \\
& \lambda_{a} \mathbf{Y}_{1^{\prime}}^{b(c \rightarrow q)}-\zeta_{a} \mathbf{Z}_{1^{\prime}}^{b(c \rightarrow q)}
\end{aligned}
$$


The residues of the quantities introduced in Eqs. 54-59 are defined as

$$
\begin{aligned}
\mathcal{F}_{1}^{b(c \rightarrow q)} & =\mathcal{E}^{2, b}\left(\mathbf{D}^{c \rightarrow q}\right)+\mathcal{E}^{3}\left(\mathbf{D}^{b}, \mathbf{D}^{c \rightarrow q}\right), \\
\mathbf{W}^{c \rightarrow q} & =\left[\mathbf{D}^{c \rightarrow q} \mathbf{F} \mathbf{D}\right]^{\oplus}+\mathbf{D} \mathcal{F}^{c \rightarrow q} \mathbf{D}+\frac{1}{2} \omega_{q}\left[\mathbf{D}^{c \rightarrow q} \mathbf{S D}\right]^{\ominus}, \\
\mathbf{W}_{1^{\prime}}^{b(c \rightarrow q)} & =\left[\mathbf{D}^{b} \mathcal{F}^{c \rightarrow q} \mathbf{D}+\mathbf{D}^{b} \mathcal{F} \mathbf{D}^{c \rightarrow q}+\mathbf{D}^{c \rightarrow q} \mathcal{F}^{b} \mathbf{D}\right]^{\oplus}+ \\
& \frac{1}{2}\left[\omega_{b}\left(\mathbf{D}^{b} \mathbf{S} \mathbf{D}^{c \rightarrow q}\right)+\omega_{c}\left(\mathbf{D}^{c \rightarrow q} \mathbf{S}^{b} \mathbf{D}+\mathbf{D}^{c \rightarrow q} \mathbf{S} \mathbf{D}^{b}\right)\right]^{\ominus}, \\
\mathbf{Y}_{1^{\prime}}^{b(c \rightarrow q)} & =\left[\mathcal{F}^{b} \mathbf{D}^{c \rightarrow q} \mathbf{S}+\mathcal{F}^{c \rightarrow q}\left(\mathbf{D}^{b} \mathbf{S}+\mathbf{D S}^{b}\right)+\mathbf{F D}^{c \rightarrow q} \mathbf{S}^{b}\right]^{\ominus} \\
& \frac{1}{2}\left[\omega_{q} \mathbf{S}^{b} \mathbf{D}^{c \rightarrow q} \mathbf{S}\right]^{\oplus}+\frac{1}{2}\left(\omega_{b}+\omega_{q}\right)\left(\mathbf{S D}^{c \rightarrow q} \mathbf{S}^{b}\right)^{\oplus}, \\
\mathbf{Z}_{1^{\prime}}^{b(c \rightarrow q)} & =\left[\mathbf{D}^{b} \mathbf{S D}^{c \rightarrow q}+\mathbf{D}^{c \rightarrow q} \boldsymbol{S}^{b} \mathbf{D}\right]^{\oplus} .
\end{aligned}
$$

Another formulation of the quadratic response function can be set up using $k=0$ and $n=2:^{5}$

$$
\begin{gathered}
\langle\langle A ; B, C\rangle\rangle_{\omega_{b}, \omega_{c}} \stackrel{T r}{=} \mathcal{E}^{0, a b c}+\mathcal{E}^{1, a b} \mathbf{D}^{c}+\mathcal{E}^{1, a c} \mathbf{D}^{b}+\mathcal{E}^{1, a} \mathbf{D}^{b c}+\mathcal{E}^{2, a}\left(\mathbf{D}^{b}\right) \mathbf{D}^{c} \\
-\mathbf{S}^{a} \mathbf{W}^{b c}-\mathbf{S}^{a b} \mathbf{W}^{c}-\mathbf{S}^{a c} \mathbf{W}^{b}
\end{gathered}
$$

with the perturbed energy-weighted density matrix

$$
\begin{aligned}
\mathbf{W}^{b c}= & {\left[\mathbf{D}^{b c} \mathbf{F D}+\mathbf{D}^{b} \mathcal{F}^{c} \mathbf{D}+\mathbf{D}^{b} \mathbf{F} \mathbf{D}^{c}\right]^{\oplus}+\mathbf{D} \mathcal{F}^{b c} \mathbf{D}+\frac{1}{2} \omega_{b}\left[\mathbf{D}^{b} \mathbf{S}^{c} \mathbf{D}+\right.} \\
& \left.\mathbf{D}^{b} \mathbf{S} \mathbf{D}^{c}\right]^{\ominus}+\frac{1}{2} \omega_{c}\left[\mathbf{D}^{c} \mathbf{S}^{b} \mathbf{D}+\mathbf{D}^{c} \mathbf{S} \mathbf{D}^{b}\right]^{\ominus}+\frac{1}{2}\left(\omega_{b}+\omega_{c}\right)\left[\mathbf{D}^{b c} \mathbf{S D}\right]^{\ominus},
\end{aligned}
$$

for which also a residue can be formed

$$
\begin{gathered}
\lim _{\omega_{c} \rightarrow \omega_{q}}\left(\omega_{c}-\omega_{q}\right)\langle\langle A ; B, C\rangle\rangle_{\omega_{b}, \omega_{c}} \stackrel{T r}{=} \mathcal{E}^{1, a} \mathbf{D}^{b(c \rightarrow q)}+\mathcal{E}^{1, a b} \mathbf{D}^{c \rightarrow q}+\mathcal{E}^{2, a}\left(\mathbf{D}^{b}\right) \mathbf{D}^{c \rightarrow q} \\
-\mathbf{S}^{a} \mathbf{W}^{b(c \rightarrow q)}-\mathbf{S}^{a b} \mathbf{W}^{c \rightarrow q},
\end{gathered}
$$


using the intermediate quantity

$$
\begin{aligned}
\mathbf{W}^{b(c \rightarrow q)}= & {\left[\mathbf{D}^{b(c \rightarrow q)} \mathbf{F} \mathbf{D}+\mathbf{D}^{b} \mathcal{F}^{c \rightarrow q} \mathbf{D}+\mathbf{D}^{b} \mathbf{F} \mathbf{D}^{c \rightarrow q}+\mathbf{D}^{c \rightarrow q} \mathcal{F}^{b} \mathbf{D}\right]^{\oplus}+\mathbf{D} \mathcal{F}^{b(c \rightarrow q)} \mathbf{D}+} \\
& \frac{1}{2} \omega_{b}\left[\mathbf{D}^{b} \mathbf{S} \mathbf{D}^{c \rightarrow q}\right]^{\ominus}+\frac{1}{2} \omega_{q}\left[\mathbf{D}^{c \rightarrow q} \mathbf{S}^{b} \mathbf{D}+\mathbf{D}^{c \rightarrow q} \mathbf{S D}^{b}\right]^{\ominus}+\frac{1}{2}\left(\omega_{b}+\omega_{c}\right)\left[\mathbf{D}^{b(c \rightarrow q)} \mathbf{S} \mathbf{D}\right]^{\ominus}
\end{aligned}
$$

The formulation using $k=0$ and $n=2$ contains no terms depending on Lagrangian multipliers, but instead it depends on second-order perturbed parameters. The choice of rule can in some circumstances drastically affect the size of the calculation, particularly when one or more of the perturbations are size-extensive (e.g. geometrical perturbations).

In eq 68 , we note that there are the nested density matrix residues $\mathbf{D}^{b(c \rightarrow q)}$. These density matrices are obtained from perturbed parameters of second order. For details we refer to eq 20 and ref 5,6. The single residue of a doubly perturbed perturbation parameter is

$$
\mathbf{X}^{b(c \rightarrow q)}=\lim _{\omega_{c} \rightarrow \omega_{q}}\left(\omega_{c}-\omega_{q}\right) \mathbf{X}^{b c}=\left(\mathbf{E}^{[2]}-\left(\omega_{b}+\omega_{q}\right) \mathbf{S}^{[2]}\right)^{-1} \mathbf{M}_{\mathrm{RHS}}^{b(c \rightarrow q)},
$$

with the right-hand-side vector $\mathbf{M}_{\mathrm{RHS}}^{b(c \rightarrow q)}$ formulated using residues of first-order parameters

$$
\begin{aligned}
\mathbf{M}_{\mathrm{RHS}}^{b(c \rightarrow q)} & =\lim _{\omega_{c} \rightarrow q}\left(\omega_{c}-\omega_{q}\right) \mathbf{M}_{\mathrm{RHS}}^{b c} \\
& =\left[\mathbf{F} \mathbf{D}_{P}^{b(c \rightarrow q)} \mathbf{S}+\mathcal{F}^{b} \mathbf{D}^{c \rightarrow q} \mathbf{S}+\mathcal{F}^{c \rightarrow q}(\mathbf{D S})^{b}+\mathbf{F D}^{c \rightarrow q} \mathbf{S}^{b}\right]^{\oplus}- \\
& \frac{1}{2}\left[\omega_{q} \mathbf{S}^{b} \mathbf{D}^{c \rightarrow q} \mathbf{S}+\left(\omega_{b}+\omega_{q}\right)\left(\mathbf{S D}_{P}^{b(c \rightarrow q)} \mathbf{S}+\mathbf{S D}^{c \rightarrow q} \mathbf{S}^{b}\right)\right]^{\oplus}
\end{aligned}
$$

The particular contribution to the density matrix $\mathbf{D}_{P}^{b(c \rightarrow q)}$ is

$$
\begin{gathered}
\mathbf{D}_{P}^{b(c \rightarrow q)}=\mathbf{D S}(\mathbf{D S})^{b} \mathbf{D}^{c \rightarrow q}+\mathbf{D S D}^{c \rightarrow q} \mathbf{S D}^{b}+\mathbf{D S D}^{c \rightarrow q} \mathbf{S}^{b} \mathbf{D}+ \\
(\mathbf{D S})^{b} \mathbf{D}^{c \rightarrow q} \mathbf{S D}+\mathbf{D}^{c \rightarrow q} \mathbf{S D}^{b} \mathbf{S D}+\mathbf{D}^{c \rightarrow q} \mathbf{S}^{b} \mathbf{D S D}- \\
(\mathbf{D S})^{b} \mathbf{D}^{c \rightarrow q}-\mathbf{D}^{c \rightarrow q} \mathbf{S D}^{b}-\mathbf{D}^{c \rightarrow q} \mathbf{S}^{b} \mathbf{D} .
\end{gathered}
$$

As discussed in section 2.2.2, we can extract the first-order transition moment from the 
expressions in both formulations of the single residues and therefore obtain the second-order transition moments for $k=n=1$ as

$$
\begin{aligned}
\mathcal{M}_{0 \rightarrow q}^{a b} \stackrel{\operatorname{Tr}}{=} \mathcal{E}^{1, a b} \mathbf{D}_{q}+\mathcal{E}^{2, a}\left(\mathbf{D}^{b}\right) \mathbf{D}_{q}+\mathcal{F}_{q, 1}^{b} \mathbf{D}^{a}- \\
\mathbf{S}^{a b} \mathbf{W}_{q}-\mathbf{S}^{a} \mathbf{W}_{q, 1^{\prime}}^{b}-\lambda_{a} \mathbf{Y}_{q, 1^{\prime}}^{b}-\zeta_{a} \mathbf{Z}_{q, 1^{\prime}}^{b},
\end{aligned}
$$

where the intermediates marked $q$ can be obtained by exchanging the residue of the perturbed density matrix $\mathbf{D}^{c \rightarrow q}$ by the density matrix $\mathbf{D}_{q}$.

For the choice $k=0$ and $n=2$, the second-order transition moment is

$$
\begin{aligned}
\mathcal{M}_{0 \rightarrow q}^{a b} \stackrel{\operatorname{Tr}}{=} & \mathcal{E}^{1, a} \mathbf{D}_{q}+\mathcal{E}^{1, a b} \mathbf{D}_{q}^{c}+\mathcal{E}^{2, a}\left(\mathbf{D}^{b}\right) \mathbf{D}_{q} \\
& -\mathbf{S}^{a} \mathbf{W}_{q}-\mathbf{S}^{a b} \mathbf{W}_{q}
\end{aligned}
$$

\section{References}

(1) Olsen, J.; Jørgensen, P. J. Chem. Phys. 1985, 82, 3235-3264.

(2) Christiansen, O.; Jørgensen, P.; Hättig, C. Int. J. Quantum Chem. 1998, 68, 1-52.

(3) Hättig, C.; Christiansen, O.; Jørgensen, P. J. Chem. Phys. 1998, 108, 8331-8354.

(4) Kjærgaard, T.; Jørgensen, P.; Olsen, J.; Coriani, S.; Helgaker, T. J. Chem. Phys. 2008, $129,054106$.

(5) Thorvaldsen, A. J.; Ruud, K.; Kristensen, K.; Jørgensen, P.; Coriani, S. J. Chem. Phys. 2008, 129, 214108.

(6) Friese, D. H.; Beerepoot, M. T. P.; Ringholm, M.; Ruud, K. J. Chem. Theory Comput. 2015, 11, 1129-1144.

(7) Hettema, H.; Jensen, H. J. Aa.; Jørgensen, P.; Olsen, J. J. Chem. Phys. 1992, 97, $1174-1190$. 
(8) Sałek, P.; Vahtras, O.; Guo, J.; Luo, Y.; Helgaker, T.; Ågren, H. Chem. Phys. Lett. 2003, 374, 446-452.

(9) Koch, H.; Jørgensen, P. J. Chem. Phys. 1990, 93, 3333-3344.

(10) Hättig, C.; Christiansen, O.; Jørgensen, P. J. Chem. Phys. 1998, 108, 8355-8359.

(11) Friese, D. H.; Hättig, C.; Ruud, K. Phys. Chem. Chem. Phys. 2012, 14, 1175-1184.

(12) Jonsson, D.; Norman, P.; Ågren, H. J. Chem. Phys. 1996, 105, 6401-6419.

(13) Cronstrand, P.; Luo, Y.; Norman, P.; Ågren, H. Chem. Phys. Lett. 2003, 375, 233-239.

(14) Cronstrand, P.; Norman, P.; Luo, Y.; Ågren, H. J. Chem. Phys. 2004, 121, 2020-2029.

(15) Ringholm, M.; Jonsson, D.; Ruud, K. J. Comput. Chem. 2014, 35, 622-633.

(16) Ringholm, M.; Jonsson, D.; Bast, R.; Gao, B.; Thorvaldsen, A.; Ekström, U.; Helgaker, T.; Ruud, K. J. Chem. Phys. 2014, 140, 034103.

(17) Friese, D. H.; Bast, R.; Ruud, K. ACS Phot. 2015, 2, 572-577.

(18) Hansen, A. E.; Avery, J. Chem. Phys. Lett. 1972, 13, 396-398.

(19) Pedersen, T. B.; Hansen, A. E. Chem. Phys. Lett. 1995, 246, 1-8.

(20) Pedersen, T. B.; Koch, H.; Hättig, C. J. Chem. Phys. 1999, 110, 8318-8327.

(21) Pedersen, T. B.; Koch, H. J. Chem. Phys. 2000, 112, 2139-2147.

(22) Jansík, B.; Rizzo, A.; Ågren, H. Chem. Phys. Lett. 2005, 414, 461-467.

(23) Rizzo, A.; Jansík, B.; Pedersen, T. B.; Ågren, H. J. Chem. Phys. 2006, 125, 064113.

(24) Guillaume, M.; Ruud, K.; Rizzo, A.; Monti, S.; Lin, Z.; Xu, X. J. Phys. Chem. B 2010, 114, 6500-6512. 
(25) Tinoco, I. J. Chem. Phys. 1975, 62, 1006-1009.

(26) Meath, W. J.; Power, E. A. J. Phys. B 1984, 17, 763.

(27) London, F. J. Phys. Radium 1937, 8, 397.

(28) Helgaker, T.; Jørgensen, P. J. Chem. Phys. 1991, 95, 2595-2601.

(29) Bak, K. L.; Jørgensen, P.; Helgaker, T.; Ruud, K.; Jensen, H. J. A. J. Chem. Phys. 1993, $98,8873-8887$.

(30) Pecul, M.; Ruud, K.; Helgaker, T. Chem. Phys. Lett. 2004, 388, 110-119.

(31) Hansen, A. E.; Bouman, T. D. Adv. Chem. Phys. 1980, 44, 545.

(32) Pedersen, T. B.; Koch, H.; Boman, L.; Sánchez de Meras, A. M. Chem. Phys. Lett. 2004, 393, 319-326.

(33) Pedersen, T. B.; Fernández, B.; Koch, H. J. Chem. Phys. 2001, 114, 6983-6993.

(34) Ruud, K.; Stephens, P. J.; Devlin, F. J.; Taylor, P. R.; Cheeseman, J. R.; Frisch, M. J. Chem. Phys. Lett. 2003, 373, 606-614.

(35) Gunde, K. E.; Richardson, F. Chem. Phys. 1995, 194, 195-206.

(36) Toro, C.; De Boni, L.; Lin, N.; Santoro, F.; Rizzo, A.; Hernández, F. Chemistry - Eur. J. 2010, 16, 3504-3509.

(37) Díaz, C.; Echevarria, L.; Rizzo, A.; Hernández, F. E. J. Phys. Chem. A 2014, 118, 940-946.

(38) Diaz, C.; Lin, N.; Toro, C.; Passier, R.; Rizzo, A.; Hernández, F. E. J. Phys. Chem. Lett. 2012, 3, 1808-1813. 
(39) Diaz, C.; Vesga, Y.; Echevarria, L.; Stara, I. G.; Starỳ, I.; Anger, E.; Shen, C.; El Sayed Moussa, M.; Vanthuyne, N.; Crassous, J.; Rizzo, A.; Hernández, F. E. RSC Adv. 2015, 5, 17429-17437.

(40) Bak, K. L.; Jørgensen, P.; Jensen, H. J. A.; Olsen, J.; Helgaker, T. J. Chem. Phys. 1992, 97, 7573-7584.

(41) Beerepoot, M. T. P.; Friese, D. H.; Ruud, K. Phys. Chem. Chem. Phys. 2014, 16, $5958-5964$.

(42) Ågren, H.; Vahtras, O.; Koch, H.; Jørgensen, P.; Helgaker, T. J. Chem. Phys. 1993, 98, 6417-6423.

(43) Norman, P.; Jonsson, D.; Vahtras, O.; Ågren, H. Chem. Phys. Lett. 1995, 242, 7-16.

(44) Norman, P.; Jonsson, D.; Vahtras, O.; Ågren, H. Chem. Phys. 1996, 203, $23-42$.

(45) Kutzelnigg, W. Israel J. Chem. 1980, 19, 193-200.

(46) Schindler, M.; Kutzelnigg, W. J. Chem. Phys. 1982, 76, 1919-1933.

(47) Lazzeretti, P.; Malagoli, M.; Zanasi, R. Chem. Phys. Lett. 1994, 220, 299-304.

(48) Pedersen, T. B.; Koch, H.; Ruud, K. J. Chem. Phys. 1999, 110, 2883-2892.

(49) Cheeseman, J. R.; Frisch, M. J.; Devlin, F. J.; Stephens, P. J. J. Phys. Chem. A 2000, 104, 1039-1046.

(50) Srebro, M.; Govind, N.; de Jong, W. A.; Autschbach, J. J. Phys. Chem. A 2011, 115, 10930-10949.

(51) Becke, A. D. J. Chem. Phys. 1993, 98, 5648-5652.

(52) Hehre, W. J.; Ditchfield, R.; Pople, J. A. J. Chem. Phys. 1972, 56, 2257-2261.

(53) Hariharan, P. C.; Pople, J. A. Theor. Chim. Acc. 1973, 28, 213-222. 
(54) Dunning Jr., T. H. J. Chem. Phys. 1989, 90, 1007-1023.

(55) Woon, D. E.; Dunning, T. H. J. Chem. Phys. 1994, 100, 2975-2988.

(56) Aidas, K.; Angeli, C.; Bak, K. L.; Bakken, V.; Bast, R.; Boman, L.; Christiansen, O.; Cimiraglia, R.; Coriani, S.; Dahle, P.; Dalskov, E. K.; Ekström, U.; Enevoldsen, T.; Eriksen, J. J.; Ettenhuber, P.; Fernández, B.; Ferrighi, L.; Fliegl, H.; Frediani, L.; Hald, K.; Halkier, A.; Hättig, C.; Heiberg, H.; Helgaker, T.; Hennum, A. C.; Hettema, H.; Hjertenæs, E.; Høst, S.; Høyvik, I.-M.; Iozzi, M. F.; Jansík, B.; Jensen, H. J. A.; Jonsson, D.; Jørgensen, P.; Kauczor, J.; Kirpekar, S.; Kjærgaard, T.; Klopper, W.; Knecht, S.; Kobayashi, R.; Koch, H.; Kongsted, J.; Krapp, A.; Kristensen, K.; Ligabue, A.; Lutnæs, O. B.; Melo, J. I.; Mikkelsen, K. V.; Myhre, R. H.; Neiss, C.; Nielsen, C. B.; Norman, P.; Olsen, J.; Olsen, J. M. H.; Osted, A.; Packer, M. J.; Pawlowski, F.; Pedersen, T. B.; Provasi, P. F.; Reine, S.; Rinkevicius, Z.; Ruden, T. A.; Ruud, K.; Rybkin, V. V.; Sałek, P.; Samson, C. C. M.; Sánchez de Merás, A.; Saue, T.; Sauer, S. P. A.; Schimmelpfennig, B.; Sneskov, K.; Steindal, A. H.; Sylvester-Hvid, K. O.; Taylor, P. R.; Teale, A. M.; Tellgren, E. I.; Tew, D. P.; Thorvaldsen, A. J.; Thøgersen, L.; Vahtras, O.; Watson, M. A.; Wilson, D. J. D.; Ziolkowski, M.; Ågren, H. WIREs Comput. Mol. Sci. 2013, 4, 269, doi: $10.1002 /$ wcms.1172.

(57) Friese, D. H. J. Chem. Phys. 2015, accepted.

(58) Olsen, J.; Bak, K. L.; Ruud, K.; Helgaker, T.; Jørgensen, P. Theor. Chim. Acta 1995, $90,421$.

(59) Bast, R.; Ekström, U.; Gao, B.; Helgaker, T.; Ruud, K.; Thorvaldsen, A. J. Phys. Chem. Chem. Phys. 2011, 13, 2627-2651.

(60) Shcherbin, D.; Thorvaldsen, A. J.; Ruud, K.; Coriani, S.; Rizzo, A. Phys. Chem. Chem. Phys. 2009, 11, 816. 
(61) Bast, R.; Ruud, K.; Rizzo, A.; Helgaker, T. Theor. Chem. Acc. 2011, 129, 685.

(62) Thorvaldsen, A. J.; Ruud, K.; Rizzo, A.; Coriani, S. J. Chem. Phys. 2008, 129, 164110.

(63) Shcherbin, D.; Thorvaldsen, A. J.; Jonsson, D.; Ruud, K. J. Chem. Phys. 2011, 135, 134114.

(64) Kjærgaard, T.; Jørgensen, P.; Thorvaldsen, A. J.; Sałek, P.; Coriani, S. J. Chem. Theory Comput. 2009, 5, 1997.

(65) Ruud, K.; Helgaker, T. Chem. Phys. Lett. 1997, 264, 17.

(66) Ruud, K.; Helgaker, T. Chem. Phys. Lett. 2002, 352, 533. 
For Table of Contents Use Only

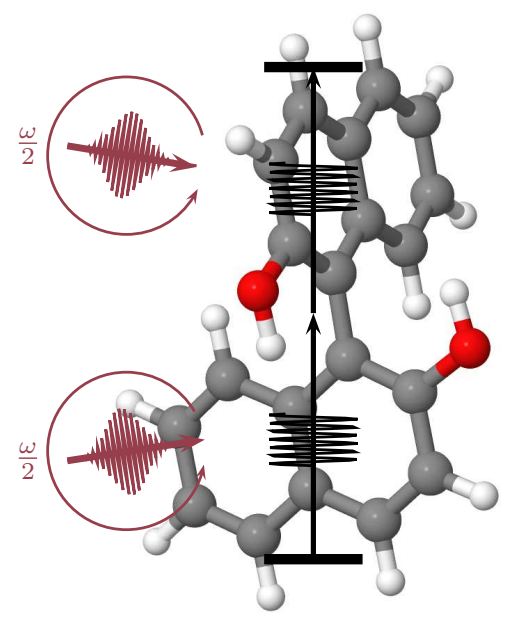

